\title{
Visual Hallucinations on Eye Closure Associated with Atropine Toxicity. A Neurological Analysis and Comparison with other Visual Hallucinations
}

\author{
C. Miller Fisher
}

\begin{abstract}
Visual hallucinations of remarkable intensity began shortly after intravenous atropine and persisted for 11 days. They were present only when the eyes were closed and were associated with heightened dreaming and disturbed sleep. The patient remained lucid and described his experiences to his attendants. Our patient's hallucinations bore some resemblance to hypnagogic hallucinations and this became the basis for the hypothesis that the hallucinations originated in the sleep-dream system of the brain stem. It is speculated that a similar site - a metabolic locus minoris resistentiae may play a part in other types of visual hallucinations and in delirium.
\end{abstract}

\begin{abstract}
RÉSUMÉ: Hallucinations visuelles les yeux clos associées à la toxicité par l'atropine. Analyse Neurologique et comparaison à d'autres hallucinations visuelles. Des hallucinations visuelles d'une intensité remarquable sont apparues peu après l'administration intraveineuse d'atropine et ont persisté pendant 11 jours. Elles étaient présentes seulement quand les yeux étaient fermés et étaient associées à une intensification des rêves et à des perturbations du sommeil. Le patient est demeuré lucide et décrivait ses expériences au personnel infirmier. Les hallucinations de notre patient avaient une certaine ressemblance aux hallucinations hypnagogiques, ce qui nous a amené à émettre l'hypothèse que les hallucinations originaient dans le système sommeil-rêve du tronc cérébral. Nous postulons qu'un site similaire - un locus minoris resistentiae métabolique pourrait jouer un rôle dans d'autres types d'hallucinations visuelles et dans le délire.
\end{abstract}

Can. J. Neurol. Sci. 1991; 18:18-27

Confusion as a result of ingesting atropine-containing flora has been recognized since ancient times. A consistent feature within the broader syndrome has been visual hallucinations which generally are not described in detail. In a recent case visual hallucinations unaccompanied by other mental manifestations followed the intravenous injection of atropine and persisted for 11 days. The patient remained lucid and described his observations to attendants who kept notes thus providing a more detailed account than usual of one individual's experience albeit in retrospect further information might have been elicited.

In seeking to define the relationship of our patient's hallucinations to other types of visual hallucinations a brief review of the subject was undertaken with the conclusion that hallucinations of the hypnagogic type appear to bear the closest resemblance to those of our patient. It is postulated that atropine toxicity acting at a locus in the brain stem associated with sleep-dream apparatus released visual hallucinations. The review became the basis for attempting to identify a somewhat similar mechanism for the other types as well. Finally it is speculated that since atropine hallucinations may simply be a mild form of atropine delirium, delirium may also be a product of a comparable disturbance within the brain stem.

\section{Case Report}

A 74 year old college-educated accountant, subject to bouts of bradycardia, received $1.0 \mathrm{mg}$ atropine sulfate intravenously and an additional $0.6 \mathrm{mg} 5$ hours later. Cardiac function then remained satisfactory but approximately 4 hours after the second dose of atropine the patient reported the onset of florid formed visual hallucinations which persisted with decreasing severity for 11 days. The patient's mouth was parched for the first 5 or 6 days making enunciation difficult and this lasted to some extent for about 10 days. For the first 3 days vision for objects closer than $35 \mathrm{~cm}$ was impaired and seeing to read returned in about 9 days. On the basis of this combination of symptoms and because no other drug was administered it was assumed that the visual hallucinations were a manifestation of atropine toxicity in a patient predisposed to an idiosyncratic reaction. The duration of the effect was longer than in any previously described case but persistence for 'several' days is not unusual.'

From the Neurology Service Massachusetts General Hospital, Boston, Massachusetts

Received June 5, 1990. Accepted September 7, 1990

Reprint requests to: Dr. C. Miller Fisher, Neurology Service, Massachusetts General Hospital, Boston, MA, USA 02114 
The patient's mind was generally lucid and he was always oriented for the date and clinical events. On awakening from a dream he would look around for things about which he had been dreaming but within 10 seconds was clear again. He was never confused. Neurological examination was normal. Visual acuity was $20 / 20$ with glasses. The visual fields were full. He was afebrile. The blood pressure was $130 / 80 \mathrm{~mm} \mathrm{Hg}$. Extensive blood tests were normal. An electroencephalogram and computerized tomographic scan were not obtained.

There was no history of a similar reaction to medication and the patient was on no long term therapy. He had once felt 'queer' and had nightmares after the use at bedtime of $10 \mathrm{mg}$ of oxycodone hydrochloride for pain. In the past haloperidol given intravenously for a minor surgical procedure was associated with mental dullness lasting about 12 hours. He was 'sensitive' to ethanolic beverages and used them sparingly. He usually slept well with little awareness of dreaming, and dreams were not colored. There was no history of eye surgery. The patient's intellect was preserved. There was no history of mental illness or depression. A sister at the age of 80 years had a pronounced but temporary post-operative delirium.

During the period of visual hallucinations the patient had vivid dreams which precluded prolonged periods of sleep and after 3 or 4 days he slept more in the daytime and nights were likely to be sleepless. While experiencing the hallucinations the patient described them to attendants who took notes. The following account is a representative sampling.

\section{The Visual Hallucinations}

Day I Four hours after the second injection of atropine sulfate, in a period of 33 minutes the patient described the hallucinations as follows: 1) panoramic country scenes; 2) an entire newspaper page of print arranged in 5 columns of approximately 500 words each, headlines and subheadlines with words, print of different sizes, all nicely arranged and presenting a good facsimile of a real page from a newspaper. On trying to read the headline by peering closely the individual letters that had given the impression of being well formed proved to be pseudo-letters, $(\Pi, \perp, !\rfloor,, \odot)$ without recognizable meaning; 3 ) the printed page of a book; 4) the page of a typed office-letter the patient had dictated 5 or 6 days before; 5) yellow and green lanes of a straightaway race track down which giant black greyhounds were running full out from left to right; 6) a large old-time cupboard about 10 feet wide with open doors and 4 shelves on which were rows of books as well as pencils, neckties and one carpet tack; 7) a giant headline KUI YANKEE; 8) animals, motor cars and village street scenes; 9) a street scene in which the patient visualized the figure of his mother who had been dead 25 years; 10) a lighthouse tower with the numbers 6808 in large size across the front; 11) a song writer's festival in Montreal the letters of whose association were GSCLP; 12) bulletin boards holding cards of many pastel colors bearing very little print at first but gradually accumulating more; 13) a blue and black poster with the letters WFIER (and the patient commented "it doesn't make sense"); 14) 7 rows of black and gray billiard balls, 6 in each row all on a billiard table; 15) large buildings including the base of the Eiffel Tower; 16) two large overlapping pumpkins and a soft felt hat; 17) some boys down town in a detective's office; 18) multiple sea shells and the patient apparently thought of blowing on a couple of conchs; 19) several magazines with brown covers and the names of various authors on the front.

Comment: The visual hallucinations came instantaneously (within a split second) upon closing the eyes and were dispelled immediately by opening the eyes. The hallucinations occurred when the patient was quite awake. When he was very tired and fell half asleep while talking, as the eyelids came down the display began and caused him to rouse. The term panoramic was often used. There was usually an overwhelming profusion of fine detail and no hallucination could be described as plain. Printed and written materials were the most frequent. The visions were vivid, nearly always of natural proportions (except possibly the big greyhounds) and often colored in pastels. There clearly was animation. The patient participated insofar as he tried to read newsprint and signs. There was never a sound and no conversation took place in the hallucinations. The visual hallucinations seemed to involve memories from all periods of the patient's past. He observed that when he was describing the hallucinations they seemed to vanish. The patient was always fully aware that the visions were not at all real. There was no associated fear, anxiety, restlessness or expression of suspicion.
Day $191 / 2$ to $12 \frac{1}{2}$ hours after atropine sulfate: 1) pages of hand written notes; 2) 100's of letters A filling a page; 3) a sheet of paper bearing the numbers 5888,$6888 ; 4$ ) a large natural waterfall (Niagara Falls?) across the brink of which was an advertisement in giant letters STILL K; 5) a traffic jam of motor cars (gridlock) extending for what was judged to be $1 / 2$ mile in all 4 directions at a cross roads; 6 ) several pages of a printed document prepared by the patient in his business office 2 days before; 7) a ham sandwich being cut into 4 pieces; 8) a man in a gray and blue outfit waiting outside a church; 9) an ant walking on a tent; 10) a picture of 5 sports figures from a newspaper; 11) dirty dishes beside a sink with a roasting pan; 12) a door the upper half of which was open to form a wicket on the shelf of which were bits of white paper; 13) an interview of participants in a research program without it being clear what exactly anyone did; 14) 5 men from a federal government agency working with another group designated by 5 letters of the alphabet; 15) 2 seaplanes arrested or impounded for entering South Boston; 16) flat cables draped over the Boston public library; 17) a series of articles in a newspaper, some strongly pro somebody, others strongly con somebody, always belligerent in tone; 18) the edge of an open front door; 19) 3 electric plugs without wires, the number 6, 2 forks and 2 flashlights.

Comments: The patient reported that waking up was very unpleasant without it being clear what form this took. When he was pressed for details of his visual hallucinations he said; "When I'm trying to figure something out it makes me sick."

Day 2 1) panoramas of small pieces of farmland of a hundred different shapes, 3 or 4 pieces of each shape and 50 different patterns per panorama with white on blue landscape dotted with black stones or boulders $30 \mathrm{~cm}$ in diameter; 2) an extremely detailed written account of directions for the construction of an ideal federal building prepared by a man and his wife; 3 ) an involved detailed printed account of various viewpoints about a business topic the patient was an expert on. He was surprised at the detailed knowledge revealed which he did not realize he had; 4) a detailed account of typed letters of correspondence from the patient's business office all couched in a florid hill-billy like dialect; 5) ten or 12 sheaves of individual papers with print easily seen for reading purposes. The writing made good sense and seemed so real that upon opening his eyes the patient sometimes asked where the sheets were.

Comments: During the first 2 days the patient saw no faces such as occur in hypnagogic hallucinations. He saw human figures but never well delineated faces. The hallucinations occurred whether the room light was on or off that is whether in light or darkness. Likewise they disappeared when the eyes were opened in the dark. They were present whether the patient was lying down or seated.

Day 3 to Day 7 Visual hallucinations of many kinds continued intermittently but they were clearly less overwhelming and less discomfitting for the patient. Each night's sleep was disturbed by unusually realistic dreams which were much more vivid than the patient's normal dreams in the past. The dreams were not frightening but were of a brilliance that startled the patient wide awake sometimes with shortness of breath. On falling asleep the same dream and the same theme recurred 10 or 12 times each night making restful sleep impossible. The patient used the terms nightmare and drivel. "These nights are wild, very complicated material, something seems to be going on all night - an old boy's reunion, building a building with words, chaos, etc." Five sedative drugs were tried in various doses on different nights - alprazolam, diphenhydramine, flurazepam, triazolam and chloral hydrate - but dreams seemed more florid and sleep was not appreciably increased. The abnormality of dreaming gradually decreased as the days passed but normality had not returned by day 8 . During this whole period the patient complained of a dry mouth and kept the comer of a wet facecloth in his mouth.

Day 8 1) flowing columns of brightly colored beads of different sizes; 2) 6 to 10 typical British soccer players in jerseys, short trousers and stockings to below the knees arranged along a line in the center of vision facing to the right - all were running furiously toward the right and as they moved along 10 became 20 , then hundreds and finally literally thousands all running until in 2 or 3 seconds they covered the surrounding hills and meadows as far as the eye could see, still running. On opening the eyes they disappeared in a flash; 3 ) hundreds of marching British soldiers in yeomanry uniforms; 4) innumerable inanimate objects not further described. 
Comment: The description illustrates the production of hundreds of duplicate images all in a second or two and dispelled in a flash by opening the eyes.

Day 10 1) while lying on his bed in daylight with the eyes closed mounds of thousands of flowing or streaming balls or rounded ornaments of many different colors. With the eyes open, while gazing at a bright surface for example the ceiling the balls of many colors would appear but on looking at a dark area they disappeared; 2) a pale-green Oklahoma oil-drilling rig appeared on the ceiling and when a technician pierced the patient's radial artery for a blood sample, the central shaft of the oil rig dipped down and came back up again. The technician had said "You'll feel a prick now" and the idea of the arterial puncture created a change in the visual hallucination conforming to the idea; 3 ) a framework of irregular squares and cubes of wire building up a huge basket work that filled multiple rooms. The 'wire' material was usually black or gray but at times was blue. "It did not move much." The "wires' were at times red and appeared to contain or represent flowing blood, the currents merging to form a network of channels all carrying flowing blood. At times the pattern of 'wires' was green resembling plant stems or vines; 4) mounds of colored glass beads, usually tiny, as if in a V-shaped container always moving downward or to the right flowing like rivers or tiny streams and at times whole mountains of beads flowed to the right. All were dispelled by opening the eyes in bright light; 5) at times the misshapen 'wire' patterns could appear on a page the patient was writing on and the page seemed to have something written on it when it really did not. Pillows and bed clothing appeared dirty, that is littered with tiny pieces of dirt but on looking more closely there was nothing there. With the eyelids closed the hallucinations could be seen through the lids if looking at a bright surface and opening the eyes dispelled them except as described in item 1. The patient wrote that during the night he had a series of different nightmares of variable content and not threatening. Each dream related to its own particular idea, a special theme suggesting on-going ideation yet each was incoherent. The themes were not a jumble but nonetheless they were ridiculous and difficult to recall. They were promptly dispelled by opening the eyes and sitting up. These dream performances were an abnormal experience for the patient except for one occasion about 10 years earlier when at bedtime he used oxycodone for brachial neuralgia. There was no normal reverie.

Day $1 /$ Visual fields filled with Christmas ornaments or decorations and in a few minutes Christmas blankets appeared colored, folded and bulging.

Comment: This was the last day that visual hallucinations were mentioned. It is remarkable that the patient's diary noted "mouth better" probably referring to dryness although at the time no association had been made between dryness of the mouth and visual hallucinations. The patient also observed that despite his exhaustion from disturbed sleep he did not recall having yawned at any time. On day 20 he noted that he yawned rather normally. Unpleasant dreams, nightmares and disturbed sleep were prominent until at least day 20. From day 3 to day 19 the patient was tired all day until 9:00 p.m. when he became wide awake for 4 or 5 hours.

\section{Summary of the Characteristics of the Visual Hallucinations}

\section{The Visual Features}

1) The hallucinations were purely visual i.e. there was no sound or other modality; 2) they were ever present, constantly changing, panoramic, vivid, realistic, of natural size, in normal perspective at appropriate distances in space, stationary or moving from left to right, seemed to involve the visual fields symmetrically and were not brightly colored; 3) the images were described as much more vivid than visual images in a dream but not as bright as normal awake vision; 4) the hallucinations were always complex and there were no elementary scintillations, lights, sparkles or stars; 5) the multiplicity of similar images (letters A, landscapes, footballers and soldiers) a phenomenon known as multiplication was noteworthy; 6 ) the images appeared instantaneously on closing the eyes and disappeared equally promptly on opening the eyes; 7) faces were not a feature of the hallucinations whereas the patient later reported that in his normal hypnagogic experiences faces were prominent; 8) the hallucinations of the first few days were highly organized and complex while in later days they were much less elaborate, fragments of wire or 'dirt' being among the last phenomena; 9) the hallucinations gradually decreased and ceased about day 11 at approximately the same time as dryness of the mouth was relieved; 10) the torrent of variegated hallucinations succeeding one another several times a minute was distinctive and represents an addition to recorded neuropsychological experience.

\section{The Independence of the Images}

11) the different items and themes visualized were overwhelmingly abundant, 'a flood', but images never repeated themselves at least in the first 7 or 8 days; 12) the images followed one another in sequences that were totally haphazard, at least no order was recognized; 13) the images were a series of discontinuous animated pictures or scenes without the continuity of a coherent narrative. Their duration was a few seconds, probably never more than 10 seconds.

\section{The Subject's Role}

14) the patient always appreciated the unreal nature of the hallucinations; 15) during the period of the hallucinations there was preservation of alertness memory, intellect, emotional composure, judgment, speech, motion, sensation and spontaneity indicating intact neocortical function; 16) the patient remembered many of the hallucinations weeks later indicating that memory function was preserved during the actual hallucinatory experience; 17) the hallucinations of papers, books and notes were clearly borrowed from the patient's professional activities of the previous month or two but footballers, soldiers, greyhounds, billiard balls and farmland had no recognizable recent memory connotation, and were probably from many decades in the past; their occurrence was not related to the season of the year; 18) hallucinations of printed material also occurred during brief periods of dozing off and were associated with the idea that the documents were meaningful and could be read with good or even brilliant sense, that is there was intellectual participation. It seemed the print came first and the illusion or delusion of meaning followed but events passed so rapidly that they could well have represented a single experience embracing all elements virtually simultaneously. It was on such occasions that on awakening he would look for and try to handle things he had been dreaming of, suggesting a possible link between dream-imagery and the hallucinations of eye-closure and thus a relationship with sleep; 19) the patient was a spectator and the hallucinations were extrinsic to his own mental processes which seemed to proceed independently although the hallucinations preempted 'attention'; 20) when the patient while awake peered closely at what seemed to be meaningful print, the letters became 'hieroglyphics' and what appeared to be specks of dirt on a page also vanished under scrutiny; 21) in the hallucination of an oil rig tower on day 10 it was remarkable that without being conscious of it, the patient's mental image of the downward movement of the needle penetrating the radial artery evoked a downward movement of the main drilling apparatus. Ideation of which the patient was unaware influenced conscious ideation. This must be evidence of central integration rather than the hallucination 
being an isolated purely visual phenomenon; 22) the hallucinations of printed material carried an emotional tone in that the newspaper articles were strongly pro or con somebody always with some belligerence. Also some of the printing gave the impression of having a hill-billy quality. Again the purely visual hallucination was accompanied by or more accurately, combined with an emotion; 23) abnormalities of dreaming consisted of increased vividness, unpleasantness without fear or anxiety, repeated perseveration of the same dream theme, prevention of the required amount of sleep and one night recurrent apnea 2 to 4 minutes after falling asleep causing the patient to waken with alarm and startle; 24) the 'runaway' hallucinations did not occur during sleep or dreaming; 25) visual acuity, allowing for impaired accommodation was preserved.

\section{Another Similar Case}

A case like the one just presented could not be found in the literature on either anticholinergic toxicity or visual hallucinations. ${ }^{2-5}$ The effect of eye-closure on hallucinations is frequently remarked on without further comment. This point will be discussed a little later. The patient of Rozanski6 noted visual hallucinations on eye-closure but changes in the patient's personality complicated the illness which otherwise had many features in common with that of the present patient.

The records of the Stroke Service of the Massachusetts General Hospital contained notes on a patient whose symptoms were remarkably similar. A 68 year old man 2 days after cardiac surgery began to have "the most remarkable vivid visual hallucinations" when he closed his eyes. He was not sleepy whatsoever and did not consider himself dreaming in the slightest. He would close his eyes and describe the 'visions' to his wife who was sitting with him. He saw pastoral scenes, street scenes, gardens, motor cars, paintings, nuns, bishops, 'floozies', and at times individual people, one a woman with a lot of hair. The figures were definitely moving. People were of normal size and life-like and their faces seemed sad. There was no sound. "It was as if the wall of the room was a huge panoramic screen." Everything was brightly colored. The shiny leaves of plants showed very fine shades of green. Flowers were of the most delicate shades of pink and every other color. "It was a thing of beauty." The door to his room had a cylinder and plunger type of airbrake for gentle closing and when the patient looked in that direction and closed his eyes the apparatus turned into a man's face with hair standing straight up clearly resembling Mikoyan the Russian ambassador to the U.S.A. The undersurface of the cylinder moved vigorously like a mouth opening and closing as if Mikoyan was speaking animatedly. On opening his eyes the 'vision' was gone. The patient had received 4 injections of atropine pre- and intra-operatively.

There were records of several other patients who experienced visual hallucinations on eye closure but the descriptions were too fragmentary for further consideration.

\section{The Nature of The Hallucinations}

\section{Types of Pure Visual Hallucinations}

Ophthalmopathic Hallucinations - associated with visual impairment resulting from disease of the ocular globes, optic nerves or optic tracts. Known as the syndrome of Charles Bonnet; here the term Ophthalmopathic will be used.
Hemianopic Hallucinations - Associated with a hemianopia (or lesser visual field defect) involving visual cortex. Visual hallucinations of migraine belong here.

Peduncular Hallucinations - Resulting from lesions of the midbrain, pons or thalamus.

Drug, Metabolic, Toxi-infective or acute Abstinence Encephalopathy.

Associated with hallucinogens - (mescaline, lysergic acid diethylamide).

\section{Narcolepsy}

Hypnagogic (Hypnopompic) Hallucinations - Occur in the process of falling asleep (or awakening).

Temporo-Occipital seizures

Isolation, sleep deprivation, sensory deprivation, fatigue, horror and hypnosis.

The Psychoses (schizophrenia, depression)

Reverie - The visual imagery of reverie is probably allied to dreaming but it is claimed visions can occur without any element of sleep.

Miscellaneous - in dementia, post seizure, nighttime imagery difficult to separate from dreaming, and other etiologies not included above.

It should be pointed out that in the first 4 types only a small percentage of patients exhibit hallucinations, leading to the conclusion that the conditions are necessary for the hallucinations but insufficient in themselves. ${ }^{2}$

Our patient's hallucinations according to the present interpretation were the result of atropine toxicity and therefore fall in the category - drug encephalopathy. A broader question here is how our patient's hallucinations in their clinical features compare with other types and where in the field of visual hallucinations our patient's syndrome finds its appropriate place. Such a comparison might well provide information concerning the mechanism of visual hallucinations in general. With these goals in mind the various kinds of visual hallucinations will be reviewed looking for a model for our patient's experience. First however it might be helpful to recount the long list of clinical details that a full description of visual hallucinations might entail. This is not readily available in the literature yet it is of special importance in a largely phenomenological study of this kind.

\section{The Description of Visual Hallucinations in General}

Starting with the basic outline of Burgermeister et al ${ }^{7}$ a broad enquiry concerning the hallucinatory experience will cover many particulars which here are divided into 4 groups: The visual features, the temporal aspects, cognitive and emotional factors, and kinds of imagery.

The visual aspects - content, theme, clarity, static or moving (intrinsic or extrinsic), direction of movement, manner of disappearance, color, vividness, complexity, size (normal, magnified, lilliputian, dwarf), form (natural, deformed or absurd) spatial properties, projection, transformation (sudden or a gradual metamorphosis), reduplication or multiplication, autoscopy, presence of sleep paralysis, number of different images, variation, white cloth effect, the effect of medical manipulation, constancy of silence, precipitating factors, lateralization and localization, the effect on vision for normal objects, relation to the surroundings, change in size with distance, effect of eye closure, 
conditions affecting the hallucinations (normal use of vision, ocular movements particularly saccades, ${ }^{8}$ time of day, brightness of lighting, noise, close scrutiny of the imagery, caloric stimulation of the vestibular system and displacement of the eyeball with the finger).

The temporal aspects - onset, evolution, overall duration, individual duration, number of images per hour or day, rate of succession, temporal relation of the hallucinations to the causal condition and the time of day.

The influence of eventide in the evocation of visual hallucinations is frequently mentioned. The following terms are found in the literature: twilight, dusk, late afternoon, toward evening, early evening, evening, the approach of night, vesperal, crepuscular, end of the day, nightfall, hour of sleep, preparatory to sleep, bed time, at night, retiring to sleep but before closing the eyes, threshold of sleep and so on. When possible the descriptions should be more precise with, for example, information concerning the exact hour, the season, the condition of the lighting in the room, whether at home or in the hospital, if the patient is in bed or not, in the act of going to sleep or not, eyes open or closed, drowsy or alert, confused, whether the patient is left undisturbed, and if there is any relation to insomnia or the occurrence of dreaming. Episodic momentary drowsiness may not be easily discerned. All of these points also apply to the problem of 'sundowning', the increase in symptoms shown by confused patients late in the day.

Cognitive and emotional factors - credulity, reaction of the subject to the hallucinations, details of the mental state, state of the memory, the presence of elements of a delirium or of sensory modalities other than visual, the influence of thought, the presence of intrinsic 'meaning' in written or printed material, dream elements, the effect of fatigue, distraction or complete relaxation, the effect of focussing attention on other subjects, visual or non-visual and interaction with the hallucinations. Insight has many shades and varies from one session of hallucinations to another. Hallucinations may merge imperceptibly with illusions or delusions. Because hallucinations are wholly subjective and beyond objective verification it is important where feasible to unobtrusively observe the patient's spontaneous behavior and reactions to the hallucinations apart from the examination.

The kinds of imagery - The practicability with which the preceding lines of enquiry can be pursued will, of course, vary according to the content of the imagery whose variety is unending. The occurrence of almost every conceivable being or thing has been recorded and in comparing cases matching content may be an important criterion. From relatively plain forms geometric patterns, crosses, networks, mosaics, ribbons, beads, balls, balloons, chains, ropes and cylinders - they range through tapestries, draperies, scarves, rugs and wallpaper. In the most complex hallucinations the commonest are human figures, singly, in groups or even multitudes, and animals of every kind, size and color. People are often fashionably dressed in brilliantly colored clothing or uniforms perfect in every detail - men, women, children and soldiers. Not uncommonly the figures are lilliputian, dwarf or misproportioned. The animals include dogs, cats, horses, cattle, lions, tigers, elephants and many others. Very frequently there are beautiful scenes of panoramic landscapes featuring the countryside, forests, parks, fields, mountains and streams. Also commonly reported are faces, pictures, portraits, bust-like figures, beautiful colored flowers, buildings, furniture, flags, handkerchiefs, parcels, birds of all kinds, insects, snakes, fish and so on. Living creatures are usually in motion suggesting activation of a memory trace. As would be expected the items have changed over the past 200 years. Rarely mentioned are writing, newsprint, food, eating utensils and police.

A complete case study should include medical, neurologic and ophthalmological examinations, enquiry re drugs, metabolic investigation, electroencephalography (sleep polygraphy), computerized tomographic imaging, magnetic resonance imaging, proton emission tomography (or single photon computed emission tomography) and finally pathologic examination.

In referring to the various kinds of mental activity involved in hallucinations and their near neighbors, dreaming and delirium, as well as in ordinary thought it is convenient to have a general designation that encompasses all of these mental transactions and for this the term ideation will be adopted here. In the normal waking state ideation consists of visual and auditory impressions that pass so swiftly and faintly as to almost evade introspection. In purely visual hallucinations the succession of images is usually slow or even static except perhaps for our patient whose hallucinations followed one another in rapid sequence. In dreaming to which hallucinatory activity has been likened the flow of imagery is relatively slow compared with awake ideation; in delirium it is probably slower still. That artificiality attends such oversimplification is attested to by the reminder that these processes are the outward signs of electrochemical changes constantly being propagated at different velocities up to $15 \mathrm{~m} / \mathrm{sec}$ and involving 2 billion neurons. In Sherrington's words - "a sparkling field of rhythmic flashing points with trains of travelling sparks hurrying hither and thither". 9 In 2 or 3 seconds of ideation nerve impulses may have travelled the length and breadth of the brain some 50 times creating a dynamic steady state.

Comparison of our patient's hallucinations with other types - In most cases of hallucinations reported in the literature many of the descriptive details enumerated above are missing and the usefulness of a precise comparison may be limited. Nonetheless each will be considered in turn. Hypnagogic hallucinations appear in the first few seconds of the passage into sleep as the subject lies with eyes closed. ${ }^{10}$ Generally the imagery is not profuse and is limited to a few items. Images of things recently viewed during the preceding daytime may appear at night on closing the eyes as a replay phenomenon ${ }^{11}$ and are regarded by some as distinct from true hypnagogic imagery which is not so related. 12 Our patient's hallucinations had several features in common with the hypnagogic type, namely the presence of eyeclosure, a relationship with sleep in that during the period of hallucinations our patient had excessive dreaming and disturbed sleep, and thirdly some of our patient's hallucinations contained actions and 'meaning' suggesting elements of dreaming. Our patient subsequently watched for his hypnagogic hallucinations and observed nothing resembling the present illness. They occurred infrequently and consisted mostly of faces often distorted and always unfamiliar.

In dozing off for a minute or even less, normally, visual imagery may appear that qualifies for dreaming rather than hypnagogic hallucinations although precise criteria for such a differentiation might be difficult to define. According to Hobson ${ }^{13-15}$ 
dreaming involves mainly the visual sensory mode but commonly auditory and tactile modes as well. There is a scene, actors and a narrative plot with a theme. An event is occurring. The dreamer is an almost invisible participant. The action is fictive and any train of thought would have to be classed as delusional. The ideation which is highly complex is characterized above all by bizarreness. Obviously the heightened visual activity in our patient bears little or no resemblance to full scale dreaming. Although dreaming is clearly hallucinatory it does contain narrative action that stamps it as dreaming. For this reason it was omitted from the foregoing list of causes of visual hallucinations.

In the past 70 years much has been learned about the ideation associated with Narcolepsy but the types of imagery are not always clearly distinguished. The vivid dream-like fantasies that occur in sleep paralysis (and waking-up paralysis) and the dream-like ideation reported by narcoleptics wakened from a sleep onset REM state are frequently referred to as hypnagogic hallucinations ${ }^{16}$ while the ordinary hypnagogic hallucinations of falling asleep are seldom mentioned. Again the distinction between dream and hallucination is undefined. Lhermitte ${ }^{17}$ emphasized the parallel between narcoleptic hallucinations and peduncular hallucinosis although the patterns of imagery are quite different.

The visual hallucinations of occipito-temporal epilepsy are usually stereotypical taking the same form repeatedly in each seizure. Cortical stimulation elicits these complex visual forms only if the patient has epilepsy. In our patient an electroencephalogram (EEG) was not obtained, but there is no reason to suspect seizure activity. As for hallucinogens our patient's hallucinations had none of the 16 characteristics of lysergic acid diethylamide (LSD) flash backs described by Abraham. 18

There remain for comparison ophthalmopathic, hemianopic and peduncular hallucinations. For this purpose a review was undertaken of 74 cases with adequate details, from the literature and from the records of the Massachusetts General Hospital. In all, there were 20 cases of ophthalmopathic hallucinations, 32 cases of hemianopic hallucinations and 22 cases of peduncular hallucinations. Seven cases in which visual hallucinations followed vertebral angiography 6,19 were kept separate rather than being included in the peduncular hallucination group.

It became evident that although great variations existed between these 3(4) types, there was considerable overlap and over all they had much in common in regard to kinds of imagery, coloring, number of items, frequency, size (lilliputian), distortion, occurrence of autoscopy, and the effect on the hallucinations of various maneuvers. Of particular importance to the present discussion, the hallucinations of our patient readily fitted into the same common mould. Two inferences might be made: 1) a common mechanism (or related mechanisms) may be responsible for the 3(4) types being discussed and for the hallucinations of our patient as well; and 2) the visual system has a limited repertoire of responses to these different kinds of insult.

If any one of the 3(4) types differed importantly from the others it was the peduncular which on average had fewer items, pastoral scenes were lacking, the hallucinations in several instances came on in the evening or when the patient was drowsy and mental lucidity or insight was compromised. Nonetheless in one case which in the literature is referred to as peduncular in type following vertebral angiography ${ }^{6}$ the clinical picture very closely resembled that of our patient and warrants a brief presentation here. The patient after the inadvertent injection of contrast agent into her right vertebral artery at angiography developed florid visual hallucinations beginning a few hours later with an assortment of geometric patterns on closing her eyes. Then for more than 10 days on closing her eyes at bedtime preparatory to sleep she saw the most beautiful colored scenes from nature a flock of flying storks, flower gardens, a tree in which sat a single bird, a winter snow scene, green pastures, a stream cascading down a mountain side and so on. On opening her eyes the imagery disappeared. She underwent a striking change of personality. Because the hallucinations occurred at the hour of sleep and were presumably related to a brain stem insult the author classified the case as one of peduncular hallucinosis although in none of the other peduncular cases reported in the literature was eye-closure mentioned as a precipitating factor. It is possible that the visual cortex was the site of the disturbance rather than the brain stem but in one of Hauge's cases of visual hallucinations following vertebrobasilar angiography 19 both posterior cerebral arteries arose from the carotid arteries and apparently the contrast agent did not reach the visual cortex.

Although peduncular hallucinations are a well recognized type it might be preferable to include them in a broader category - brain stem hallucinations that would encompass not only peduncular cases proper but also cases in which visual hallucinations are associated with pontine hemorrhage 20 thalamic hemorrhage ${ }^{21}$ and thalamic infarction. ${ }^{23}$ It might be noted in passing that de Morsier ${ }^{2}$ claimed that thalamic lesions can be associated with visual hallucinations that are similar to those encountered with occipital lobe lesions.

\section{The role of eye-closure}

One of the main features of our patient's hallucinations was their appearance on eye-closure and prompt disappearance on opening the eyes even in the dark. Eye-closure is often mentioned in the literature on hallucinations but no rules for its effect have been discerned. Eye-closure combined with some aspect of sleep or eventide normally precipitates hypnagogic hallucinations and as described brought on hallucinations in Rozanski's case. ${ }^{6}$ Hallucinations that occur with the eyes open may be abolished, diminished or augmented by eye-closure.

Bonnet's subject saw hallucinations with his eyes open or closed and equally with the left eye in which he was blind and with the right eye which retained some vision. ${ }^{24}$ Abell ${ }^{25}$ who was blind (ophthalmopathic hallucinations) saw his imagery with the eyes open or closed as did Flournoy's patient. 26 Of the 16 patients of Weinberger and Grant ${ }^{27}$ (ophthalmopathic hallucinations) 2 saw them only when the eyes were open and 2 only when the eyes were closed. Bartlett's 28 complicated patient (ophthalmopathic hallucinations) probably had hallucinations only when the eyes were open in the light. Of 4 personal cases of ophthalmopathic hallucinations one patient saw birds and vicious tigers only when relaxing with his eyes closed. A second had them only when the eyes were open and the two others with eyes open or closed. Ormond's patient ${ }^{29}$ (hemianopic hallucinations) experienced his hallucinations only when his eyes were open except for faces which were seen with the eyes open or closed. Of 2 personal patients with hemianopic hallucinations 
one could experience the imagery in the daytime with the eyes open but it was most prominent with the eyes closed preparatory to sleep. The other patient's imagery was present only in the daytime with the eyes open.

Our patient was certain that his hallucinations occurred only once when his eyes were open and that time while he was looking at a bright surface. When the lids closed in drowsiness hallucinations occurred behind the half-closed lids. Eye-closure while awake obviously excludes light but that may not be its only effect for closing the eyes implies relaxation or resting an important element in the process of decreasing arousal or promoting sleep. In that respect eye-closure could relate to an associated activity in the brain stem bearing on sleep. If eye-closure served only to exclude light the hallucinations should have continued in the dark (or under the bed covers) ${ }^{7}$ when the eyes were open. Hypnagogic hallucinations clear on opening the eyes in the dark but this is not a simple matter as the act of deliberately opening the eyes interrupts the process of falling asleep. No reference could be found of a study of the effect on the EEG of opening and closing the eyes in the dark; there are rare reports of eyeclosure in the dark provoking EEG seizure activity. ${ }^{30}$

More investigations might have been undertaken in our patient. The effect of closing one eye at a time was not tested. Hallucinations were apparently not associated with spontaneous blinking. The patient was not asked to deliberately close his eyes either completely or in graded fashion in order that the triggering process be analyzed. The effect of passively raising or lowering one or both of the patient's lids should have been tested. The effect on the EEG of having the patient close and open his eyes in the dark or after blind-folding him in the light should have been observed.

The dominant theory concerning the pathogenesis of ophthalmopathic and hemianopic hallucinations holds that they are 'release phenomena' resulting from visual impairment. 28,31 This of course is but the expression of a principle without addressing the process and mechanism. Our patient's hallucinations would qualify for the same mechanism since they were released instantaneously on the exclusion of light. There is again the objection that they did not persist in the dark with the eyes open. If ophthalmopathic and hemianopic hallucinations are a release phenomenon it might be anticipated that eye-closure would enhance them still further but this is not recorded with any regularity. Also Lamy, ${ }^{32}$ Lance $^{33}$ and Kömel ${ }^{8}$ have pointed out that hemianopic hallucinations do not appear at the same time as the brain lesion but only after an interval of delay varying from 12 hours to one week implying that a reactionary process was occurring possibly involving neurotransmitter up or down regulation. Furthermore the hallucinations are temporary and cease after a few weeks even though the hemianopia persists. Eye-closure in our patient would seem to have little in common with that kind of 'release' phenomenon. Just how eye-closure acts remains unclear but a subtle relationship to the sleep process remains a possibility.

It should be added however that a delay in the onset of hallucinations does not occur with all types of structural lesions. Brain Stem (peduncular) hallucinations for example may come on immediately after the ictus. ${ }^{23,34}$ In a personal case a patient who had undergone surgical shunting for hydrocephalus was returned to his hospital room complaining of seeing mice running around.
Examination showed paralysis of vertical gaze upward and downward. A CT scan showed the tip of the shunt tubing lying within the tegmentum of the midbrain. The tubing was retracted immediately whereupon the visual hallucinations and vertical gaze palsy were alleviated.

\section{The effect of tiredness, eventide and retiring to sleep}

Our patient's hallucinations occurred in full daylight but they were more numerous late in the day when the patient was tired and recumbent. This effect which is frequently mentioned in accounts of other types of visual hallucinations may be important for it suggests a close connection between visual hallucinations and the sleep-wake process, an association that may be relevant when developing a theory concerning their mechanism.

Ophthalmopathic hallucinations are regularly influenced by eventide according to Burgermeister et al. ${ }^{7}$ Abell's ${ }^{25}$ multitudinous hallucinations (ophthalmopathic type) came, on first retiring to bed and on several occasions when he awakened in the night (hypnopompic hallucinations). Bartlett's ${ }^{28}$ patient (ophthalmopathic type) experienced some of his hallucinations at night immediately before going to sleep but before closing his eyes. Hemianopic hallucinations have a similar association. In case 2 of Engerth et al ${ }^{35}$ the imagery was present on retiring and closing the eyes. The patient of Brust and Behrens ${ }^{36}$ noted her hallucinations nightly while she watched the television screen. In a personal case the hallucinations were mostly present with the eyes closed preparatory to sleep. However no such relationship was described in 2 large series of hemianopic hallucinations. 8,33

In the earliest stage of delirium tremens visual hallucinations may appear on closing the eyes while going to sleep and disappear on opening the eyes. In fully developed delirium tremens the hallucinations are seen with the eyes open but are enhanced by darkness and closing the eyes. The hallucinations that followed vertebrobasilar angiography occurred only after retiring preparatory to sleep. 6

In some cases an even closer relationship with sleep was noted. For example Abell25 (ophthalmopathic hallucinations) at first dreamed of beautiful landscapes and at a later date they began to appear when he was awake. In one of Terson's ${ }^{37}$ cases (ophthalmopathic hallucinations) the hallucinations ceased and were replaced by unusual nightmares. Lhermitte's ${ }^{38}$ original patient (peduncular) slept during the day and was awake at night, and the author made nocturnal enhancement of symptoms a central feature of his theory that peduncular hallucinations are an emancipated fragment of dreaming. Our patient during the period of hallucinations had unusually vivid unpleasant dreams and his day-night sleep pattern was reversed. Nightmares and sleep disturbances may not be common however for apart from these few examples they are not mentioned in the literature.3,7,29.39

\section{The mechanism of our patient's hallucinations}

In the foregoing presentation a possible relationship between our patient's hallucinations and the sleep process was remarked on from time to time. The hallucinations while prominent during the day were more florid in the evening and when the patient was tired. Hallucinations appeared when the lids drooped in drowsiness. Also the patient's sleep was disturbed, dreams were abnormally abundant and vivid, and some of the hallucinations 
had features that on close scrutiny resembled dreaming. Insofar as our patient's hallucinations were present on eye-closure, consisted of multiple unrelated visual items and occurred in the absence of damaging brain lesions, they resembled hypnagogic hallucinations more closely than any of the other types described. If the hypnagogic model is valid a link with the sleep process would be strengthened.

This is not the occasion to consider in detail how modern concepts of the organization of sleep function in the brain stem both REM and non-REM apply to the present interpretation. Hypnagogic ideation although part of the sleep process is not known to be a function of the REM system nor has that point been investigated but in all likelihood a relationship exists. Using Hobson's model13-15 one might speculate that the hypnagogic-like hallucinations of our patient were the result of hyperactivity in the REM activating nucleus in the pontine reticular formation or in its effector system whose activity subserves or permits dreaming. A weakness in this formulation is that Hobson's REM activating center is cholinergic and one would expect therefore that atropine would inhibit REM activity rather than evoking hyperactivity. As an alternative to REM participation it might be noted that non-REM dreaming is common in early sleep ${ }^{40,41}$ and it is possible that atropine lowered the threshold not only for dream imagery but also for hypnagogic hallucinations. If the hypnagogic nature of our patient's hallucinations that at times bordered on dreams can be substantiated a relationship with REM function would become a virtual certainty. Perhaps the most that can be claimed at this time is that our patient's hallucinations bore a relation to sleep function and hence may represent a metabolic abnormality in the brain stem.

In the description of the patient's illness it was emphasized that his mentation was intact and his hallucinations were highly complex indicating that neocortical function must have been preserved and that the impetus for the abnormal ideation should be sought elsewhere than in the cortex. The preceding speculation is in keeping with this. Regarding this principle, Jackson ${ }^{42}$ wrote: "a delirious patient does not 'see a rat' by aid of diseased structures any more than an aphasic swears by aid of the softened part of the brain". It might be added parenthetically that Jackson speculated that the symptoms of atropine poisoning were secondary to disturbances of respiration and circulation and that action on the visual centers of the cerebral hemispheres need not be supposed. 43

\section{The mechanism of the other visual hallucinations discussed herein}

Having traced the origin of our patient's hallucinations to a disturbance within the sleep-wake system of the brain stem we now turn to a brief consideration of the larger question - the mechanism of the other kinds of visual hallucinations described above. It will be recalled that the comparative study revealed that the several types had sufficient features in common to suggest they shared a common underlying mechanism. In most instances a disturbance in the visual system would be suspect but in the case of peduncular hallucinations the lesions clearly lay outside what is considered to be the visual domain and in pursuing the theme of a common mechanism the challenge would be to elucidate a brain stem mechanism to explain hypnagogic, ophthalmopathic and hemianopic hallucinations.
Hypnagogic hallucinations being related to sleep would pose no problem and a central locus of action is assumed. Ophthalmopathic hallucinations are greatly influenced by relaxation and somnolence bespeaking a tie with the sleep process. This is not necessarily a strong point however since a disturbance of ideation could be modified by sleep function and yet have a separate origin. Ophthalmopathic hallucinations are frequently so elaborate and perfect that a well-functioning cortex must be available. Moreover they are visualized straight ahead rather than being lateralized. The direction of the horizontal movement of the images is of unknown significance.

Hemianopic hallucinations are visualized only in the impaired field and disappear if they move past the center of vision. It is a generally held view that hemianopic hallucinations are 'released' in the visual association area as a result of a lesion of the primary visual area of the occipital lobe analogues to the 'release' of ophthalmopathic hallucinations associated with blindness. 33 It would appear that here a peripheral or local mechanism must be operative but there is the reservation that brain stem-cortical ascending activity is a regular participant in ideation both in sleep and awakeness and this would be an exception. Furthermore the hallucinations are inhibited by saccades ${ }^{8}$ an action that is thought to have a central mechanism. Although often elaborate the hallucinations are purely visual and this would be unexpected of a non-convulsive process originating in the visual association area. Rarely a relationship with sleep and fatigue has been described. ${ }^{35}$ Lesions of the optic tract and optic radiations have not been reported as a cause of hemianopic hallucinations. It may be speculated that brain stem-cortical impulses are disturbed or modified at the cortical level and a commonality of mechanism with the other types of hallucinations would be preserved. However at this time the matter must be regarded as unsettled.

In summary, just as in the case of our patient's hallucinations the evidence has been marshalled in support of a central or brain stem mechanism involving the sleep-dream apparatus, for hypnagogic, ophthalmopathic and hemianopic hallucinations using peduncular hallucinations as the prototype. Another hypothetical common mechanism would be one more closely tied to the visual system but against this would be the various points advanced in favour of a central process.

This formulation is in many respects an expansion or generalization of Lhermitte's 17 explanation of peduncular hallucinations as a dissociation in the sleep system whereby dream images are released in the presence of slight drowsiness. Lhermitte regarded peduncular hallucinations as a 'narcoleptic equivalent', a classic instance of dreaming while awake. That the ideation described in narcoleptic dreaming is a complex narrative rather than separate unassociated images was not discussed. It is of interest that in one of Lhermitte's peduncular cases the hallucinations at a late stage became hypnagogic. Use of the narcoleptic model was probably inaccurate and a connection with the hypnagogic process might be more appropriate.

\section{EXTRAPOLATION}

Visual hallucinations occur regularly in atropine toxicity in humans, usually combined with delirium and confusion.! This association is such a constant feature that the visual hallucinations 
of our patient may be regarded as the earliest stage of the more severe syndrome, the fortuitous suspension of the process at a point critical to the generation of hallucinations. Atropine delirium would simply represent a more advanced, a quantitatively greater dysfunction within the sleep system whence the ideation of dreaming is normally derived. This is far from a radical suggestion since for centuries physicians have regarded delirium as a waking dream (Morton 1694). ${ }^{44}$ That view has probably been based on the plain fact that of all the facets of human behavior it is dreaming that most closely resembles the behavior of delirium. Expressed in neurological terms, it is only in dreams and hallucinations (delirium) that the brain creates realistic ideational patterns that conform more or less to external experience. It is difficult to obtain more convincing evidence that a bonafide relationship exists. In brief the proposition being offered here is that the acute toxic encephalopathy of atropine also has its genesis in the brain stem neural system related to sleep. The disturbance would represent hyperexcitation in the REM system and inhibition of sleep.

Speculation may be carried a step even further. It is well known from clinical experience that the various delirious states have many features in common. As Wolf and Curran ${ }^{45}$ emphasized regardless of the underlying cause the clinical picture tends to be stereotyped and diagnostic interpretation cannot be based on symptomatology alone. If this be true it follows that what has been said about atropine delirium may hold for acute delirious states in general namely that they originate in the same brain stem neural apparatus which can be construed as a biochemical or metabolic locus minoris resistentiae. The term delirium is used here to refer in particular to hypervigilant confused states including the delirium tremens of acute abstinence, major postoperative confusion, acute infections with fever and some cases of acute drug intoxication. In delirium focal cortical deficits are not obvious a finding consistent with the hypothesis that the locus of dysfunction is elsewhere than in the cortex, that is, in the brain stem.

Barbiturates and alcohol two drugs commonly associated with withdrawal delirium both have their preferred site of action in the brain stem. ${ }^{46,47} \mathrm{In}$ animals tolerance of barbiturates is associated with an increase in the number of acetylcholine receptors in the brain stem in which state withdrawal symptoms would represent a relative insufficiency of acetylcholine analogous to the action of atropine in our patient.

The means to test this hypothesis is at present largely limited to clinical analysis. Delirium, hallucinations, dreaming and sleep are certainly intermixed but demonstrating that the locus of the interlocking process is in the brain stem is another matter. A cogent question is why brain stem hemorrhage, infarction and tumor, infrequently cause delirium (personal observation). Possibly structural damage is too gross an insult for in delirium the brain is minimally and reversibly deranged. In pursuing the general thesis polygraphic evaluation of the hallucinating individual could provide information about REM activity. Medical manipulation of REM to determine the effect on the hallucinations would be another approach.

\section{ACKNOWLEDGEMENTS}

This study was supported by the Mannie and Ruth Cohen Foundation, Washington, D.C.

\section{REFERENCES}

1. Goodman LS, Gilman A, eds. The Pharmacological Basis of Therapeutics, 5th Edition. New York: MacMillan, 1985; 572.

2. de Morsier G. Les hallucinations. Étude oto-neuro-ophtalmologique. Rev Oto-neuro-ophtal 1938; 16: 241-352.

3. Hécaen H, Badaraco JG. Semeiologie des hallucinations visuelles en clinique neurologique. Acta Neurol Latinoamer 1956; 2: 23-57.

4. West LJ, ed. Hallucinations. New York and London: Grune \& Stratton, 1962

5. Duke-Elder WS. Text-Book of ophthalmology, Vol. 12. St. Louis: C.V. Mosby Company, 1971.

6. Rozanski J. Peduncular hallucinosis following vertebral angio-graphy. Neurology 1952; 2: 341-349.

7. Burgermeister $\mathbf{J}$, Tissot $\mathrm{R}$, de Ajuriaguerra J. Les hallucinations visuelles des ophtalmopathes. Neuropsychologia 1965; 3: 9-38.

8. Kölmel HW. Complex visual hallucinations in the hemianopic field. J Neurol Neurosurg Psychiatry 1985; 48: 29-38.

9. Sherrington CS. Man on his nature, 2nd Edition. New York: Doubleday \& Company, 1953.

10. Maury MA. Des hallucinations hypnagogiques ou des erreurs des sens dans l'état intermédiare entre la veille et le sommeil. Ann Médico-Psychol 1848; 11:26-40.

11. Fisher CM. Neurologic fragments II. Remarks on anosognosia, confabulation, memory and other topics; and an appendix on selfobservation. Neurology 1989; 39: 127-132.

12. Vihvelin H. On the differentiation of some typical forms of hypnagogic hallucinations. Acta Psychiat Neurol 1948; 23: 359-389.

13. Hobson JA. The dreaming brain. New York: Basic Books, 1988.

14. Hobson JA, McCarley RW. The brain as a dream state generator. An activation-synthesis hypothesis of the dream process. Amer $J$ Psychiat 1977; 134: 1335-1348.

15. Hobson A. Sleep mechanisms and pathophysiology: some clinical implications of the reciprocal interaction hypothesis of sleep cycle control. Psychosomatic Med 1983; 45: 123-140.

16. Hishikawa $Y$, Kaneko Z. Electroencephalographic study on narcolepsy. Electroencephalogr Clin Neurophysiol 1965; 18: 249-259.

17. Lhermitte J. Désordre de la fonction hypnique et hallucinations. Ann Médico-Psychol 1938; 1: 1-14.

18. Abraham HD. Visual phenomenology of the LSD flashback. Arch Gen Psychiat 1983; 40: 884-889.

19. Hauge T. Catheter vertebral angiography. Acta Radiologica Suppl 1954; 109: 1-219.

20. Nakajima K. Clinicopathological study of pontine hemorrhage. Stroke 1983; 14: 483-493.

21. Fisher CM. The pathologic and clinical aspects of thalamic hemorrhage. Trans AM Neurol Assoc 1959; 84: 56-59.

22. Chavany JA. Referred to by de Morsier. ${ }^{2}$

23. Feinberg WM, Rapcsak SZ. 'Peduncular hallucinosis' following paramedian thalamic infarction. Neurology 1989; 39: 1535-1536.

24. Flournoy H. Le cas de Charles Bonnet: Hallucinations visuelles chez un vieillard operé de la cataracte. Arch Psychol Suisse 1901; 1: $1-23$.

25. Abell T. Remarkable case of illusive vision. Boston Med Surg 1845; 33: 409-413.

26. Flournoy $\mathrm{H}$. Atypical lilliputian hallucinations in an old man with cataract. Encephale 1923; 18: 566-579.

27. Weinberger EA, Grant FC. Visual hallucinations and their neurooptical correlates. Arch Ophthalmol 1940; 23: 166-199.

28. Bartlett JEA. A case of organized visual hallucinations in an old man with cataracts and their relation to the phenomenon of the phantom limb. Brain 1951; 74: 363-373.

29. Ormond AW. Visual hallucinations in sane people. Br Med J 1925; 2: 376-378.

30. Vignaendra V, Loh TG, Lim CL, et al. Epileptic discharges triggered by blinking and eye closure. Electroencephal Clin Neurophysiol 1976; 40: 491-498.

31. Cogan DG. Visual hallucinations as release phenomena. von Graefes Arch Klin Ophthalmol 1973; 188: 139-150.

32. Lamy H. Hemianopsie avec hallucinations dans la partie abolie du champ de la vision. Rev Neurol 1895; 3: 129-135. 
33. Lance JW. Simple formed hallucinations confined to the area of a specific visual field defect. Brain 1976; 99: 719-734.

34. Geller TJ, Bellur SN. Peduncular hallucinosis: Magnetic resonance imaging confirmation of mesencephalic infarction during life. Ann Neurol 1987; 21: 602-604.

35. Engerth $G$, Hoff $H$, Pötzl $O$. Zur patho-physiologie der hemianopischen Halluzinationen. Ztschr Ges Neurol Psychiat 1935; 152: 399-421.

36. Brust JCM, Behrens MM. "Release Hallucinations" as the major symptom of Posterior Cerebral Artery Occlusion: a report of 2 cases. Ann Neurol 1977; 2: 432-436.

37. Terson A. Hallucinations visuelles chez des ophtalmopaths. Ann d'oculiste 1930; 167: 815-825.

38. Lhermitte J. Syndrome de la calotte de pedoncule cerebral. Les troubles psycho-sensoriels dans les lesions du mesoncephale. Rev Neurol 1922; 29: 1359-1365.

39. Baruk MH. Les hallucinations visuelles. Paris: Bull Soc Opthal, 1936; 8: 713-739.

40. Maron L, Rechtschaffen A, Wolpert EA. The sleep cycle during napping. Arch Gen Psychiat 1964; 11: 503-508.
41. Foulkes D, Vogel G. Mental activity at sleep onset. J Abnorm Psychol 1965; 70: 231-243.

42. Jackson $H$. The factors of insanities. Medical Press and Circ 1894; 57: 615-619.

43. Jackson JH. Selected Writings of Hughlings Jackson. Taylor J, Homes $G$ and Walshe FMR, eds. New York: Basic Books, 1958; 2:421.

44. Mettler CC. History of Medicine; A correlative text, arranged according to subjects. Philadelphia Blakiston 1947; 552.

45. Wolff HG, Curran D. Nature of delirium and allied states. Arch Neurol Psychiat 1935; 33: 1175-1215.

46. Tabakoff B, Munoz-Marcus M, Fields JZ. Chronic ethanol feeding produces an increase in muscarinic cholinergic receptors in mouse brain. Life Sci 1979; 25: 2173-2180.

47. Nordberg A, Wahlstrom G, Larsson C. Increased number of muscarinic binding sites in brain following chronic barbiturate treatment to rat. Life Sci 1980; 26: 231-237. 